\title{
Detailed Unification Equations for Kinetic Parameters: A Case Study on Human Salivary Alpha Amylase
}

\begin{abstract}
Background: There has been an equation which unifies all common kinetic parameters to the exclusion of pseudo-first order rate constant. Much attention had been paid to rate constant for the production of reducing sugars during amylolysis catalyzed by alpha amylase. There seems not be concern for the rate $\left(\boldsymbol{k}_{2[S]}\right)$ of amylolysis of glycosidic bond and making of bonds. Modification of active site for therapeutic and scientific reasons can alter the value of $\boldsymbol{k}_{2[\mathrm{~s}]}$.
\end{abstract}

Objectives: The objectives of this research are the formulation of an additional, simple, and verifiable mathematical models that can be used to determine the parameter, exp ( $k t)$ and $\Delta\left[S_{0}\right]$ given $\left[S_{0}\right]$ where $k, t$ and $\left[\mathrm{S}_{0}\right]$ are the pseudo-first order rate constant, duration of assay and concentration of the substrate and, more importantly, the equation for the determination of $\boldsymbol{k}_{2[\mathrm{~S}]}$.

Method: The theoretical aspect entailed the derivation of different equations while the experimental aspect entailed the application of Bernfeld method of enzyme assay; this was used to determine the molar concentration of reducing sugar produced.

Results: Unpaired $t$ - test showed that the usual or conventional equations gave quantitative results that were not statistically different $(P>0.05)$ from the quantitative results from the derived equations in this research. The value of $\boldsymbol{k}_{2[s]}$ was $9202.74 \pm 119.65 / \mathrm{min}$.

Conclusion: Unification quadratic equations were derived and give quantitative results similar to the results obtained from conventional equations. The value of $\boldsymbol{k}_{2[S]}$ $\sim 1 / 2^{\text {nd }}$ of $k_{2}$; this shows that hydrolysis of glycosidic bond and making of new bonds is the rate limiting step. Estimation of the duration of assay needed to produce a desired amount of reducing sugar may be for feature investigation.

Keywords: Rate constant for product formation; Rate constant for the hydrolysis of glycosidic bond and bond making; Pseudo-first order rate constant; MichaelisMenten constant; Maximum velocity of hydrolysis; Quadratic equations; Degree of polymerization

Research Article
Volume 6 Issue 4 - 2017
Ilkechulkwu Iloh Udema1,2*
${ }^{1}$ Research Division of Ude International Concepts Limited (RC
862217) B.B. Agbor, Delta State, Nigeria
Biochemistry and Chemistry Department.
${ }^{2}$ Owa Alizomor Sec Sch, Owa Alizomor, Ika North East, Delta
State, Nigeria.
*Corresponding author: Ikechukwu Iloh Udema, Research
Division of Ude International Concepts Limited (RC 862217)
B.B. Agbor, Delta State, Nigeria,Biochemistry and Chemistry
Department, Owa Alizomor Sec Sch, Owa Alizomor, Ika North
East, Delta State, Nigeria.
Email: udema_ikechukwu99@yahoo.com
Received: October 25, 2017 | Published: November 20,
2017

\section{Introduction}

There has been lot of interest in different kinds of rate constant which characterizes different enzymes [1-5]. Most enzymes studied may be described as industrial enzymes because of commercial objectives, which include production of industrial fuels, bioethanol [6-8], production of bulking agent, water retention agent, thickeners etc [4]. Some of the products are highly important in a wide range of nutritional, cosmetic and pharmaceutical applications [10-12]. The hydrolysis of starch from various sources is widely studied [12-16]. Most of the industrial enzymes studied are usually mesophiles and most often, thermophiles of microbial origin [17]. This research focuses mainly on a polymer called potato starch and human salivary alpha amylase which, has similar capacity and function as pancreatic amylase. It is not a misplacement to use salivary amylase (EC 3.2.1.1) as a model for this study because its normal activity is evidence of good health. It is a maker for various health indices $[18,19]$. It has been observed that direct entry of starch into the small intestine, by-passing partial digestion in the oral cavity results in substantially less starch digestion in the small intestine and glucose absorption [20]. These observations make it worthwhile to treat human salivary amylase exclusively.

Unlike polysaccharides whose degree of polymerization $\phi$ (the number of glucose molecules in a molecule of a polysaccharide) are quite large requiring much longer duration of assay on the assumption that the polysaccharide is totally amenable to total amylolysis, the hydrolysis of sugar whose $\phi$ ranges from 2-5, as pointed in a submitted manuscript [21], is much more straightforward. "For instant if $n$ moles of maltose are totally hydrolyzed, $2 n$ moles of glucose should be yielded; thus the rate constant for the production of glucose molecules is twice the rate constant for the hydrolysis of maltose"[21].

As in a parallel paper, submitted elsewhere, using Aspergillus oryzea alpha amylase as an enzyme model, this research is not concerned with mechanism of enzyme catalyzed reaction, but such mechanism cannot be detached from the rate of formation of the desired and/or any by product. However, most often the rate 
of formation of the product is the focus to the exclusion of the rate of the process or the mechanistic steps to give the product. The mechanism by which glycosidic bond is hydrolyzed is a process which requires time $[22,23]$. This view and the fact that it may be a rate limiting step, has been expressed in a submitted paper treating another homologue. Therefore, so long as challenges such as need for energy for worthwhile purpose, athleticism/labour and health challenge such as diabetics which requires regulation of blood glucose level are common experiences, it is instructive to consider as part of the objectives of this research the formulation of an additional, simple, and verifiable mathematical models similar to Eq. (1a) anchored on basic principle that can be used to determine the parameter, exp $(k t)$ and $\Delta\left[S_{0}\right]$ given $\left[S_{0}\right]$ where $k, t$ and $\left[\mathrm{S}_{0}\right]$ are the pseudo-first order rate constant, duration of assay and concentration of the substrate and, more importantly, for the determination of the rate of hydrolysis of glycosidic bond, the duration of which is part of the total time taken to yield the product.

The significance of the intended objectives is better appreciated if cognizance is taken of the fact that it is the hydrolysis of the bond that is the rate limiting step; this is the reason while thermophiles in particular are less active at low temperatures due to low conformational flexibility of the active site domain unlike psychrophiles whose cold temperature environment does not inhibit its activity because its active site domain is already in a state of conformational flexibility, eliminating the need for higher temperature dependence $[17,24]$. Apart from swallowing starchrich diet, by-passing partial digestion in oral cavity as suggested elsewhere [16,20], a complex process of gene transplant coding for amylase with lower capacity to hydrolyze glycosidic bond or ingestion of capsules encapsulating such enzyme may also enhance the control of digestion and plasma sugar level in diabetics. Extract from natural sources, Moringa oleifera [25] may also aide the control of diabetics, though it is not certain whether amylase is susceptible to direct and indirect effect of the extract at its active site.

\section{Formulation of unification equations-the theory}

Rate constant in particular, for most enzymes, hydrolases in particular, are regularly studied for different reasons. The most studied is the pseudo-rate constant which according to Butterworth et al. [1] may not be accurate on the basis of the mathematical formalism applied in its determination and in particular, the rate constant otherwise called turnover number [23]. The rate constant equation, (Eq. (1a)) below is another expression which is often cited by astute and higher mathematical biologist [26,27] as shown below. According to Tzafrifri [26] and, Schnell and Maini [27], the equation was original work of Goldstein [28] and Cha et al. [29].

$$
v=\frac{k_{2}}{2}\left(\left(K_{m}+\left[E_{0}\right]+[\check{\mathrm{S}}]\right)-\sqrt{\left(K_{m}+\left[E_{0}\right]+[\check{\mathrm{S}}]\right)^{2}-4[\check{\mathrm{S}}]\left[E_{0}\right]}\right)
$$

Where $v, k_{2}, K_{\mathrm{m}}$, and $\left[E_{0}\right]$ are the velocity of hydrolysis of starch, rate constant for the production of reducing sugar maltose, Michaelis-Menten constant, and the concentration of the enzyme respectively; The parameter $[\check{S}]$ expresses the sum of the mass concentration of free substrate and substrate involved in complex formation with the enzyme [27]: $[\check{S}]=[S]+[C]=\left[S_{0}\right]-[P][27]$ where $[S],\left[S_{0}\right],[C]$, and $[P]$ are the concentration of free substrate, total concentration of substrate, concentration of enzymesubstrate complex, and concentration of product respectively. The attraction for the equation is initial unfamiliarity and for its inspiring capacity for application to answer or proffer solution to another question vis-à-vis barrage of criticism against linear plot for the determination of kinetic parameters [30].

The issue regarding the equation is that Michaelis-Menten constant $\left(K_{m}\right)$ and $[\check{S}]$ must be in mol/L. Consequently the molar mass of the substrate must be known in order to maintain dimensional consistency. However, the view that “ $\left(K_{m}\right)$ "gives information about the proportion of enzyme present as enzymesubstrate complex (ES) as well, but for the reaction at steadystate, i.e. while the enzyme is actually 'at work', busy in turning over substrate" [31] may be examined with caution. If $K_{\mathrm{m}}$ remains the substrate concentration at half maximum velocity $\left(v_{\max }\right)$ and $v_{\max } / k_{2}=\left[\mathrm{E}_{0}\right], v_{\max } / k_{2} / 2 \neq K_{\mathrm{m}} / M_{\mathrm{s}}$ where $M_{\mathrm{s}}$ is the molar mass of the substrate. Otherwise, $\left(v_{\max } / k_{2} / 2\right)^{-1} K_{\mathrm{m}}=M_{\mathrm{s}}$ which may not be the case, if for instance maltose is the substrate. Besides, hydrolysis of glycosidic bond in sugars whose degree of polymerization » 2 occurs one after the other coupled with dissociation of the complex leaving behind a fragment and maltose, the product, for instance, after each hydrolytic action.

A complex equation such as Eq. (1a) unlike the usual or conventional simple expression such as $v_{\max } /\left[E_{0}\right] \equiv v\left(K_{\mathrm{m}}+\left[S_{0}\right]\right) /\left[S_{0}\right]$ $\left[E_{0}\right]$ is far more encompassing bringing relational link between variables which characterize the activity of an enzyme with element of conservation principle despite paucity in its use. This may not belie its usefulness as supported by Baici's view that "A classic paper is not necessarily one that has been, and continues to be, cited at a high rate. It can also be one that marked a starting point and stimulated the growth of an entire branch of science" [31].

Once again as in a submitted manuscript [21] in which a different homologue is addressed the following equations below lead to some quadratic equations that may serve as a unifying equation for different variable including in particular, pseudo-rate constant, $k$, for the change in the concentration of the substrate with progress in amylolysis. In line with intellectual honesty and integrity it is imperative to state that Eq. (1) through Eq. (22) had been derived in another manuscript treating another homologue of alpha amylase of microbial origin [21]. It is repeated in this research so as to preclude doubt regarding the basis or origin of Eq. (22) below.

$$
\frac{\Delta\left[S_{0}\right]}{M_{x}}(\phi-1) N_{A}=\text { Number of glycosidic bonds }\left(b_{G}\right)
$$

Where $\phi,\left[S_{0}\right], N_{\mathrm{A}}$, and $M_{\mathrm{x}}$ is the degree of polymerization, mass concentration of the substrate, Avogadro's number, and molar mass of the substrate if the entire chain of a polysaccharide 
is hydrolyzed otherwise it can best be described as the molar mass of that part of the substrate hydrolyzed, which ensures dimensionless parameter, $b_{\mathrm{G}}$. The basis of Eq. (1) is that if the degree of polymerization of a polysaccharide is $\phi$, and $M_{\mathrm{x}}$ is the molar mass of the part of the substrate hydrolyzed, the number of glycosidic covalent bonds should be $\phi-1$. However, for the purpose of this investigation, a simple expression for the total number $(\phi)$ of glucose molecule is given as:

$$
\begin{gathered}
\phi=\frac{M_{\mathrm{x}}}{162} \\
\Delta\left[S_{0}\right]=\left[S_{0}\right]\left(1-e^{-k t}\right)
\end{gathered}
$$

Where $k$ and $t$ are the pseudo-first order rate constant for the utilization of the substrate and duration of assay. Equation (3) is derivable from first principle [32] as follows: $[S]=\left[S_{0}\right] e^{-k t}$ such that $\Delta\left[S_{0}\right]=\left[S_{0}\right]-\left[S_{0}\right] e^{-k t}$. Marchal et al. [33] determined pseudo-first order rate constant taking into cognizance mass conservation law $\left[\mathrm{mmol} /\left(\mathrm{kg} \mathrm{dw}+\mathrm{H}_{2} \mathrm{O}\right)\right]$. The authors [33] recognized the addition of water to the hydrolytic process. In this paper, however, the number of bonds that can be hydrolyzed given appropriate hydrolase is $\phi-1$ where, $\phi$ is as earlier defined. There is need to add that with human salivary alpha amylase only some of the bonds need to be hydrolyzed. For convenient sake exp $(k t)$ for $e^{k t}$ and $\exp (-k t)(1 / \exp (k t))$ for $e^{-k t}$ are to be used subsequently.

Equations (1b) and (3) show mass concentration of the substrate converted to product. Consequently, if the mass of the product is implied in line with mass conservation law division by the molar mass of the product, if certain gives the number of moles per unit volume. In this case $\phi$ should be the degree of polymerization of the product. On account of this, the following relationship may hold:

$$
[P] N_{A}=\frac{\beta\left[S_{0}\right]}{M_{3}}\left(1-\frac{1}{\exp (k t)}\right)\left(\frac{M_{3}}{162}-1\right) N_{A}
$$

Where $\boldsymbol{\beta}$ may be referred to as a proportionality constant if $M_{3}$ is taken as the molar mass of the product, maltose. It may be necessary to point out that Eq. (3) and its appearance in Eq. (4) is from the expression as explained earlier from first principle.

Equation (4) is premised on the fact that for every mole of maltose yielded, one mole of the glycosidic bond is hydrolyzed. This is equivalent to the loss of two glycosidic bonds from the polysaccharide. Rearrangement and elimination of the parameter $N_{\text {A }}$ gives:

$$
[P]=\frac{\frac{\beta\left[S_{0}\right](\exp (k t)-1)}{\exp (k t)}\left(\frac{M_{3}}{162}-1\right)}{M_{3}}
$$

Rearrangement of Eq. (5) gives:

$$
\frac{\left[S_{0}\right]}{162}-\frac{\left[S_{0}\right]}{M_{3}}=\frac{\exp (k t)[P]}{\beta(\exp (k t)-1)}
$$

$$
\frac{\left[S_{0}\right]}{M_{3}}=\frac{\left[S_{0}\right]}{162}-\frac{\exp (k t)[P]}{\boldsymbol{\beta}(\exp (k t)-1)}
$$

Let Eq. (8a) below holds temporarily for the purpose of brevity.

$$
\begin{gathered}
\zeta=\left[S_{0}\right] \frac{1}{162}-\frac{\exp (k t)[P]}{\boldsymbol{\beta}(\exp (k t)-1)} \\
M_{3}=\frac{\left[S_{0}\right]}{\zeta}
\end{gathered}
$$

It is necessary to bear in mind that the reducing sugar produced within specified duration of assay is not necessarily from one polysaccharide. Just as $k_{2}$ is expressed as $v_{\max } /\left[E_{0}\right]$, where $k_{2}, v_{\max }$ and $\left[E_{0}\right]$ are the rate constant for the production of reducing sugar, maltose for instance, maximum velocity of the production of the reducing sugar, maltose in this case, and the molar concentration of the enzyme, the equivalent rate constant, $k_{2[S]}$ (not a pseudorate constant) for the transformation of a given amount of the substrate, being equal to the mass of the product in line with conservation law is:

$$
k_{2[S]}=\frac{\Delta\left[S_{0}\right] k_{2}}{v t M_{3}}
$$

The term transformation is simply the breaking and making of bonds before the departure of the product. In Eq. (9a), $\frac{v}{L}: \frac{\Delta\left[S_{0}\right]}{}$ is simply an expression of the ratio of the molar $k_{2} t M_{3} k_{2[\mathrm{~s}]}$

concentration of the enzyme involved in complex formation to the molar concentration of product obtained from the concentration of hydrolyzed starch that was transformed to product or more precisely, the molar concentration of hydrolyzed glycosidic bonds (for each glycosidic bond hydrolyzed one maltose molecule is given), with known value of $M_{3}$. Therefore, if $\Delta\left[S_{0}\right]$ is taken as mass of product, in line with mass conservation principle, then $M_{3}$ becomes the molar mass of the product - maltose for instance - and $k_{2[S]}$ should be $\cong k_{2}$. The approximation is indicated because of imperfection in every assay.

$$
=\frac{\Delta\left[S_{0}\right] k_{2} \zeta}{v t\left[S_{0}\right]}
$$

Equation (9b) is obtained by replacing $M_{3}$ with Eq. (8b). In Eq. $(9 \mathrm{a}), k_{2[\mathrm{~s}]} \alpha 1 / M_{3}$. Thus,

$$
k_{2[S]}=\frac{\Theta}{M_{3}}
$$

Where, for the purpose of brevity, $\Theta$ is $\frac{\Delta\left[S_{0}\right] k_{2}}{v t}$ Substitute Eq. (8b) into Eq. (10) to give:

$$
k_{2[\mathrm{~s}]}=\frac{\Theta \zeta}{\left[S_{0}\right]} \equiv \frac{\Theta\left(\frac{\left[S_{0}\right]}{162}-\frac{[P] \exp (k t)}{\boldsymbol{\beta}(\exp (k t)-1)}\right)}{\left[S_{0}\right]}
$$




$$
\left[S_{0}\right] k_{2[S]}=\Theta \frac{\left[S_{0}\right]}{162}-\frac{\Theta[P] \exp (k t)}{\beta(\exp (k t)-1)}
$$

If $k_{2[\mathrm{~s}]}$ is replaced by Eq. (9a) the result is:

$$
\left[S_{0}\right] \Delta\left[\Delta S_{0}\right] \frac{k_{2}}{v t M_{3}}=\Theta \frac{\left[S_{0}\right]}{162}-\frac{\Theta[P] \exp (k t)}{\beta(\exp (k t)-1)}
$$

Rearrangement of Eq. (13) gives:

$$
\begin{aligned}
& {\left[S_{0}\right] \Delta\left[S_{0}\right] \frac{k_{2}}{v t}=\Theta\left[S_{0}\right] \frac{M_{3}}{162}-\frac{\Theta[P] \exp (k t) M_{3}}{\boldsymbol{\beta}(\exp (k t)-1)}} \\
& \Delta\left[S_{0}\right]=\frac{\left(\Theta\left[S_{0}\right] \frac{M_{3}}{162}-\frac{\Theta[P] \exp (k t) M_{3}}{\boldsymbol{\beta}(\exp (k t)-1)}\right) v t}{\left[S_{0}\right] k_{2}}
\end{aligned}
$$

Equation (9a) could be rearranged to give:

$$
v t=\frac{k_{2} \Delta\left[S_{0}\right]}{M_{3} K_{2}[\mathrm{~s}]}
$$

The purpose of Eq. (16) is the determination of a slope $\left(\boldsymbol{S}_{\mathbf{L}(1)}\right)$ which could be used to find another expression for $M_{3}$. The slope from the plot of $v t$ (or $[P])$ versus $\Delta\left[S_{0}\right]\left(\left[S_{0}\right] \exp ((k t)-1) / \exp (k\right.$ $t)$ ) can be expressed as:

$$
S_{\mathbf{L}(1)}=\frac{k_{2}}{M_{3} k_{2[\mathrm{~s}]}}
$$

Equation (17a) leads to Eq. (17b).

$$
k_{2[s]}=\frac{k_{2}}{M_{3} S_{\mathbf{L}(1)}}
$$

From Eq. (17a)

$$
M_{3}=\frac{k_{2}}{S_{\mathbf{L}(1)} k_{2[\mathrm{~s}]}}
$$

Substitution of Eq. (18) into Eq. (15) gives:

$\Delta\left[S_{0}\right]=\left(\Theta\left[S_{0}\right] \frac{k_{2}}{162 S_{\mathbf{L}(1)} k_{2[\mathrm{~s}]}}-\frac{\Theta[P] \exp (k t) k_{2}}{\boldsymbol{\beta}(\exp (k t)-1) \boldsymbol{S}_{\mathbf{L}(1)} k_{2[\mathrm{~s}]}}\right) v \frac{t}{\left[S_{0}\right] k_{2}}$

Meanwhile, $[P]=v t$ and Eq. (18) is substituted into Eq. (10) to give:

$$
\begin{aligned}
& k_{2[\mathrm{~s}]}=\frac{\Theta}{k_{2}} \boldsymbol{S}_{\mathrm{L}(1)} k_{2[\mathrm{~s}]} \\
& \Theta=\frac{k_{2}}{\boldsymbol{S}_{\mathbf{L}(1)}}
\end{aligned}
$$

Rearrangement of Eq. (19) and substitution of Eq. (21) into it gives the following after simplification,

$$
\begin{aligned}
& \Delta\left[S_{0}\right]=\Theta\left(\frac{\left[S_{0}\right] k_{2}}{162 S_{\mathbf{L}(1)} k_{2[\mathrm{~s}]}}-\frac{[P] \exp (k t) k_{2}}{\beta(\exp (k t)-1) S_{\mathbf{L}(1)} k_{2[\mathrm{~s}]}}\right) v \frac{t}{\left[S_{0}\right] k_{2}} \\
& =\frac{k_{2}}{k_{2[\mathrm{~s}]} S_{\mathbf{L}(1)}^{2}}\left(\frac{\left[S_{0}\right]}{162}-\frac{[P] \exp (k t)}{\beta(\exp (k t)-1)}\right) v \frac{t}{\left[S_{0}\right]}
\end{aligned}
$$$$
\text { As stated earlier in the text (Eq. (17b)) } \frac{k_{2}}{k_{2[\mathrm{~s}]}}=\mathrm{S}_{\mathrm{L}(1)} M_{3} \text {. }
$$

Therefore, substitution into Eq. (22) gives:

$$
\Delta\left[S_{0}\right]=\frac{S_{\mathrm{L}(1)} M_{3}}{S_{\mathrm{L}(1)}^{2}}\left(\frac{\left[S_{0}\right]}{162}-\frac{[P] \exp (k t)}{\beta(\exp (k t)-1)}\right) v \frac{t}{\left[S_{0}\right]}
$$

However, Michaelis-Menten equation can be substituted into Eq. (23) so as to replace $v$ and $[P]$ which is $\equiv v t$. The result is:

$$
\Delta\left[S_{0}\right]=\frac{M_{3}}{S_{\mathrm{L}(1)}}\left(\frac{\left[S_{0}\right]}{162}-\frac{\exp (k t)}{\beta(\exp (k t)-1)} \frac{\left[E_{0}\right] k_{2}\left[S_{0}\right] t}{\left(K_{\mathrm{m}}+\left[S_{0}\right]\right)}\right) \frac{\left[E_{0}\right] k_{2}\left[S_{0}\right] t}{\left(K_{\mathrm{m}}+\left[S_{0}\right]\right)} \frac{1}{\left[S_{0}\right]}
$$

$\frac{\Delta\left[S_{0}\right] S_{\mathrm{L}(1)}}{M_{3}}=\frac{\left[E_{0}\right]\left[S_{0}\right] t k_{2}}{162\left(K_{\mathrm{m}}+\left[S_{0}\right]\right)}-\frac{\exp (k t)}{\beta(\exp (k t)-1)}\left(\frac{\left[E_{0}\right] t}{K_{\mathrm{m}}+\left[S_{0}\right]}\right)^{2} k_{2}^{2}\left[S_{0}\right]$ In Eq. (25), $\frac{\exp (k t)}{(\exp (k t)-1)}=\frac{\left[S_{0}\right]}{\Delta\left[S_{0}\right]}$. Therefore, substitution

$\frac{\Delta\left[S_{0}\right] S_{\mathrm{L}(1)}}{M_{3}}=\frac{\left[E_{0}\right]\left[S_{0}\right] t k_{2}}{162\left(K_{\mathrm{m}}+\left[S_{0}\right]\right)}-\frac{\left[S_{0}\right]^{2}}{\Delta\left[S_{0}\right] \boldsymbol{\beta}}\left(\frac{\left[E_{0}\right] t}{K_{\mathrm{m}}+\left[S_{0}\right]}\right)^{2} k_{2}^{2}$

Rearrangement of Eq. (26) gives:

$$
k_{2}=\frac{\frac{\left[E_{0}\right]\left[S_{0}\right] t}{162\left(K_{\mathrm{m}}+\left[S_{0}\right]\right)} \pm^{2} \sqrt{\left(\frac{\left[E_{0}\right]\left[S_{0}\right] t}{162\left(K_{\mathrm{m}}+\left[S_{0}\right]\right)}\right)^{2}-4 \frac{S_{\mathrm{L}(1)}}{M_{3}} \frac{\left[S_{0}\right]^{2}}{\boldsymbol{\beta}}\left(\frac{\left[E_{0}\right] t}{K_{\mathrm{m}}+\left[S_{0}\right]}\right)^{2}}}{\frac{\left[S_{0}\right]^{2}}{\Delta\left[S_{0}\right] \boldsymbol{\beta}}\left(\frac{\left[E_{0}\right] t}{K_{\mathrm{m}}+\left[S_{0}\right]}\right)^{2}}
$$

Simplification of Eq. (27) gives:

$$
\begin{gathered}
k_{2}=\frac{\frac{1}{162} \pm^{2} \sqrt{\frac{1}{162^{2}}-4 \frac{S_{\mathrm{L}(1)}}{M_{3} \beta}}}{2 \frac{\left[S_{0}\right]}{\Delta\left[S_{0}\right] \beta} \frac{\left[E_{0}\right] t}{\left(K_{\mathrm{m}}+\left[S_{0}\right]\right)}} \\
k_{2}=\frac{\Delta\left[S_{0}\right]\left(K_{\mathrm{m}}+\left[\mathrm{S}_{0}\right]\right)\left(\frac{1}{162} \pm^{2} \sqrt{\left.\frac{1}{162^{2}}-4 \frac{S_{\mathrm{L}(1)}}{M_{3} \beta}\right)}\right.}{2\left[S_{0}\right]\left[E_{0}\right] t}
\end{gathered}
$$


Cross multiplication by $\left[E_{0}\right]$ gives $v_{\max }$ on the left hand side of the equation. But as stated earlier in the text, $\Delta\left[S_{0}\right]=\left[S_{0}\right]$ (exp $(k$ $t)-1) / \exp (k t)$. Substitution into Eq. (29) gives first:

$k_{2}=\frac{\left[S_{0}\right]\left(1-\frac{1}{\exp (k t)}\right) \beta\left(K_{\mathrm{m}}+\left[\mathrm{S}_{0}\right]\right)\left(\frac{1}{162} \pm^{2} \sqrt{\left.\frac{1}{162^{2}}-4 \frac{S_{\mathrm{L}(1)}}{M_{3} \beta}\right)}\right.}{2\left[S_{0}\right]\left[E_{0}\right] t}$

Simplification and rearrangement of Eq. (30) gives:

$$
\frac{1}{\exp (k t)}=1-\frac{2 k_{2}\left[E_{0}\right] t}{\beta\left(K_{\mathrm{m}}+\left[\mathrm{S}_{0}\right]\right)}\left(\frac{1}{162} \pm^{2} \sqrt{\frac{1}{162^{2}}-4 \frac{S_{\mathrm{L}(1)}}{M_{3} \beta}}\right)
$$

Making $\exp \left(\begin{array}{ll}k & t\end{array}\right)$ subject of the formula gives after rearrangement:

$$
\exp (k t)=\frac{\beta\left(K_{\mathrm{m}}+\left[\mathrm{S}_{0}\right]\right)\left(\frac{1}{162} \pm^{2} \sqrt{\left.\frac{1}{162^{2}}-4 \frac{S_{\mathrm{L}(1)}}{M_{3} \beta}\right)}\right.}{\beta\left(K_{\mathrm{m}}+\left[\mathrm{S}_{0}\right]\right)\left(\frac{1}{162} \pm^{2} \sqrt{\frac{1}{162^{2}}-4 \frac{S_{\mathrm{L}(1)}}{M_{3} \beta}}\right)-2 k_{2}\left[E_{0}\right] t}
$$

Knowing that $v t=[P]$, Eq. (22) can then be transformed into a quadratic equation as follows. As indicated in submitted manuscript [21] treating another alpha amylase of microbial origin, Eq. (33) below is obtained by the expansion of Eq. (22) to give:

$$
\frac{\exp (k t)-1}{\exp (k t)}\left[S_{0}\right]^{2} \frac{k_{2[S]}}{k_{2}} S_{\mathrm{L}(1)}^{2}=\left(\frac{\left[S_{0}\right]}{162}-\frac{[P] \exp (k t)}{\boldsymbol{\beta}(\exp (k t)-1)}\right)_{(33)}^{[P]}
$$

Equation (33) is as it is after cross multiplication, because $\Delta\left[S_{0}\right]=\left[S_{0}\right](\exp (k t)-1) / \exp (k t)$. Further rearrangement gives:

$\frac{(\exp (k t)-1)^{2}}{(\exp k t)^{2}}\left[S_{0}\right]^{2} S_{\mathrm{L}(1)}^{2} \frac{k_{2[S]}}{k_{2}}=\frac{\frac{\left[S_{0}\right]}{162}(\exp (k t)-1)[\mathrm{P}]}{\exp (k t)}-\frac{[P]^{2}}{\boldsymbol{\beta}}$

Let, for the purpose of simplicity, $\chi=(\exp (k t)-1) / \exp (k t)$. Thus,

$$
\chi=\frac{\left(\frac{\left[S_{0}\right]}{162}[P] \pm \sqrt{\left.\left(\frac{\left[S_{0}\right][P]}{162}\right)^{2}-4 \frac{[P]^{2}}{\beta}\left[S_{0}\right]^{2} S_{\mathrm{L}(1)}^{2} \frac{k_{2}[S]}{k_{2}}\right)}\right.}{2\left[S_{0}\right]^{2} S_{\mathrm{L}(1)}^{2} \frac{k_{2[S]}}{k_{2}}}
$$

As already stated above, $\Delta\left[S_{0}\right]=\left[S_{0}\right](\exp (k t)-1) / \exp (k t)$ such that Eq. (34a) can be rearranged to give:

$$
\Delta\left[S_{0}\right]^{2} S_{\mathrm{L}(1)}^{2} \frac{k_{2[S]}}{k_{2}}-\frac{\Delta\left[S_{0}\right][P]}{162}+\frac{[P]^{2}}{\boldsymbol{\beta}}=0
$$

$$
\Delta\left[S_{0}\right]=\frac{\frac{[P]}{162} \pm \sqrt{\frac{[P]^{2}}{162^{2}}-4 S_{\mathrm{L}(1)}{ }^{2} \frac{k_{2[S]}}{\beta k_{2}}[P]^{2}}}{\frac{2 S_{\mathrm{L}(1)}^{2} k_{2[S]}}{k_{2}}}
$$

Equations (22) and (36) are equivalents. However, Eq. (35) which leads to Eq. (36) enables the determination of $\boldsymbol{\beta}$ as follows. First is the expression as follows.

A plot of $v t$ (or $[P]$ ) versus $\Delta\left[S_{0}\right]$ in Eq. (16) $\left(M_{3}=324\right)$ gives the slope, $S_{\mathrm{L}(1)}$ which is $=k_{2} / 324 k_{2[S]}$. Therefore, $k_{2[S]} / k_{2}=1 / 324 S_{\mathrm{L}(1)}$. The implication is that $k_{2[S]}<k_{2}$ because $1 / S_{\mathrm{L}(1)}<324$ (or $\cong 324$ ). The conversion of $[P]$ to $\Delta\left[S_{0}\right]$ (i.e. $324[P]$ ) takes into account mass conservation law [33-36] and accounts for the fact that hydrolysis involves the uptake of water molecule which adds to the total weight of the product and substrate [33,34]. The substitution of the conversion, $\Delta\left[S_{0}\right] / 324$ into Eq. (35), leads to:

$$
\Delta\left[S_{0}\right]^{2} S_{\mathrm{L}(1)}^{2} \frac{k_{2[S]}}{k_{2}}-\frac{\left(\Delta\left[S_{0}\right]\right)^{2}}{162 \times 324}+\frac{\left(\Delta\left[S_{0}\right]\right)^{2}}{\beta 324^{2}}=0
$$

Simplification gives:

$$
S_{\mathrm{L}(1)}^{2} \frac{k_{2[S]}}{k_{2}}-\frac{1}{162 \times 324}+\frac{1}{324^{2} \boldsymbol{\beta}}=0
$$

Meanwhile, from the plot of $[P]$ versus $\left[S_{0}\right]$ (Eq. (16)) is the slope, $S_{\mathrm{L}(1)}=\frac{k_{2}}{M_{3} k_{2[S]}}$ (where $\left.M_{3}=2 \times 162\right)$ which upon rearrangement gives $\frac{k_{2[s]}}{k_{2}}=\frac{1}{2 S_{\mathrm{L}(1)} \times 162}$. Substitution of the latter into Eq. (38) gives, after rearrangement.

$$
\frac{S_{\mathrm{L}(1)}}{M_{3}}-\frac{1}{162 \times 324}+\frac{1}{324^{2} \beta}=0
$$

And calculation gives,

$$
\frac{1}{\beta}=0.977456
$$

It is very clear that Eq. (39) is a general one in which the parameter to be determine is the slope, $S_{\mathrm{L}(1)}$ from the plot of $[P]$ versus $\Delta\left[S_{0}\right]$.

The most important objective of this research is the determination of the rate constant for hydrolysis of a glycosidic bond or the rate at which water molecules partake in the hydrolytic action being the rate limiting step. As in submitted manuscript, the formulation of the equation requires that $\boldsymbol{\beta}$ should not appear in any of the equations. The idea is very recent (in submitted manuscript treating microbial enzyme), and for quick reference, the formulation is presented in appendix section. 


\section{Materials and Methods}

\section{Materials}

Human salivary alpha amylase was obtained from the researcher and soluble potato starch was purchased from Sigma - Aldrich, USA. Hydrochloric acid, sodium hydroxide, and sodium chloride, were purchased from BDH Chemical Ltd, Poole England. Tris, 3, 5-dinitrosalicylic acid, maltose, and sodium potassium tartrate tetrahydrate were purchased from Kem light laboratories Mumbai India, while potassium iodide was purchased from Merck Germany. Distilled water was purchased from local market.

\section{Equipment}

Electronic weighing machine was purchased from Wenser Weighing Scale Limited and 721/722 visible spectrophotometer was purchased from Spectrum Instruments China. $\mathrm{PH}$ meter was purchased from Hanna Instruments, Italy. Water bath was purchased from Hospibrand, USA.

\section{Methods}

One gram of potato starch was mixed in $100 \mathrm{~mL}$ of distilled water and boiled at $100^{\circ} \mathrm{C}$ for 3 minutes, cooled to room temperature, and decrease in volume was corrected by topping the volume with distilled water to $100 \mathrm{~mL}$ to give $16 \mathrm{~g} / \mathrm{L}$ as stock. Dilution of the stock was made to give concentrations ranging from $6-16 \mathrm{~g} /$ L. Stock solution of the enzyme was prepared by centrifuging ice-cold $20 \mathrm{~mL}$ of saliva. Centrifuged saliva was diluted with a mixture of tris - $\mathrm{HCl}$ buffer $(p \mathrm{H}=6.9), \mathrm{NaCl}(\mathrm{aq})(0.9 \mathrm{~g} / 100 \mathrm{~mL})$, $\mathrm{CaCl}_{2(a q)}(0.065 \mathrm{~g} / \mathrm{mL})$ and distilled water to give a final solution whose concentration is $1 / 2$ the concentration of stock saliva solution. A 5 minutes centrifugation was at approximately $3000 \mathrm{rpm}$ (or at 1343g). The mass concentration $(\sim 18.872 \mathrm{mg} / \mathrm{L})$ of the enzyme was determined as described elsewhere [37].

The determination of rate of hydrolysis of the glycosidic bond and making of bond is given in its simplest form as Eq (B.2). Assay was according to Bernfeld method [38] for the quantification of the molar concentration of reducing sugar, maltose and kinetic parameter, maximum velocity of hydrolysis in particular was by Lineweaver-Burk [39] and direct linear [40] plots. The value obtained from direct linear plot was adopted. The determination of rate constant for the hydrolysis of endoglycosidic bond and making of bond requires the determination of the first and second slopes: This is where assaying of the enzyme to generate data-velocity of hydrolysis with substrate concentration ranging from $6-16 \mathrm{~g} / \mathrm{L}$, maximum velocity of hydrolysis obtained from direct linear plot and via linear Lineweaver-Burk plot, and ultimately rate constant for product formation- is indispensible. The pseudo-first order rate constant $k$, was obtained from the plot of In $\left[S_{0}\right]-$ In ( $\left.\left[S_{0}\right]-324[P]\right)$ versus $t$. In order to verify the validity of the quadratic equation and any other equation the first slope from the plot of $[P]$ versus $\Delta\left[S_{0}\right]$ (Figure 1) was substituted into Eq. (22) and Eq. (34b) for the determination of $\Delta\left[S_{0}\right]$ and exp $(k t)$ respectively. The determination of rate constant for the hydrolysis of the glycosidic bond and making new bond requires a plot of $\Delta\left[S_{0}\right]$ versus $\left(\frac{\left[S_{0}\right]}{162}-\frac{[P] \exp (k t)}{(\exp (k t)-1)}\right) v \frac{t}{\left[S_{0}\right]}$ or the simplified form, $\left(\frac{1}{162}-\frac{[P]}{\Delta\left[S_{0}\right]}\right)[P]$ as shown under Figure 2. The first and second slopes are then substituted into Eq. (B.2) to give after calculation the rate constant for the hydrolysis of the glycosidic bond and making of bond.

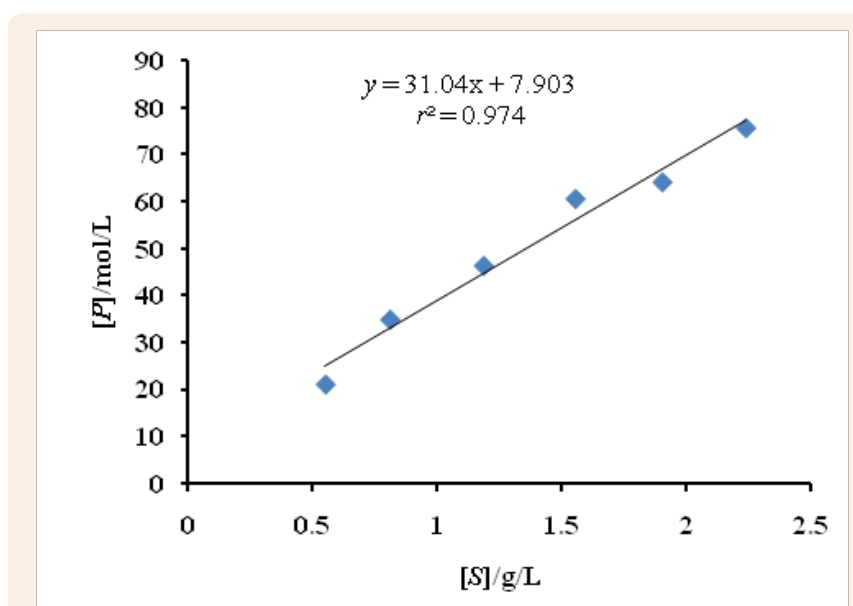

Figure 1: Plot of $v t$ versus $\Delta\left[S_{0}\right]$ for separate determination of the first $\left(S_{\mathrm{L}(1)}\right)$ of two slopes.

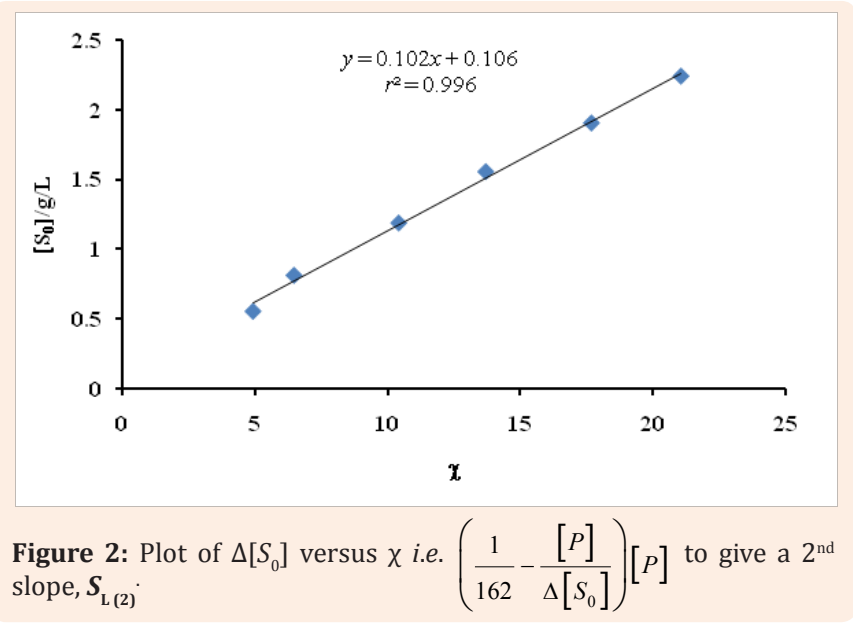

\section{Statistical analysis.}

Unpaired $t$-test for significant difference is carried out using internet based graph pad (www.graphpad. com/quick calcs / $t$-test). Micro-soft Excel was used to determine standard deviation $(n=7)$.

\section{Results and Discussion}

Various equations have been derived. Since results can be mathematical in nature or also quantitative, it is imperative to analyze the derived equations with a view to elucidate possible feature application. Only corollaries have to be highlighted and not outright re-derivation. In order to apply the unification equations unlike Eq. (1a), the only factor that should be determined is the 
factor $\boldsymbol{\beta}$ (Eq. (38-40)) which is mainly dependent on the slope from the plot of $[P]$ versus $\Delta\left[S_{0}\right]$. The motivation for this research in part as it was elsewhere is Eq. (1a) which like the mathematical results from this research contains all the kinetic parameters though the structure of the equation is partially different from all the quadratic equations in this research, Eq. (28-30). The parameters missing in Eq. (1a) is the pseudo-first order rate constant and $\Delta\left[S_{0}\right]$ unlike the derived Eq. (28-30) and other equations, Eq. (31$34 \mathrm{~b})$, for instance.

The summation $\left(K_{m}+\left[E_{0}\right]+[\check{S}]\right)$ contains different parameters namely concentrations of the remaining substrate $[\check{S}]=[S]+[C]$ (or) $\left[S_{0}\right]-[P] \quad[27]$ which has already been explained. The implication is that with a given value of $v$ and $k_{2}$, the total concentration of the substrate at zero time, free substrate $[S]$, substrate which formed complex with the enzyme $[C]$, Michaelis-Menten constant, and the molar concentration of the enzyme, the molar mass of the substrate may be determined by the rearrangement of Eq. (1a). First, $K_{\mathrm{m}^{\prime}}[\check{S}]$, $\left[S_{0}\right],[P]$, and $\left[E_{0}\right]$ should be seen to be in molar concentrations such that $[\check{S}]^{\mathrm{mc}}\left(\operatorname{or}\left[S_{0}\right]^{\mathrm{mc}}-[P]^{\mathrm{mc}}\right), K_{m}^{m c}$, should be the mass concentration of the corresponding concentration of substrate (where mc means mass concentration and not exponent or power.). Then following the rearrangement of Eq. (1a) and given that $M_{\mathrm{S}}$ is the molar mass of the substrate, then substitution of $\frac{[\check{S}]^{\mathrm{mc}}}{M_{\mathrm{S}}}$ and $\frac{K_{m}^{m c}}{M_{\mathrm{S}}}$ into Eq. (1a) should give after, expansion and rearrangement Eq. (41) below.

$$
M_{\mathrm{S}}=\frac{v\left(K_{\mathrm{m}}^{\mathrm{mc}}+[\check{\mathrm{S}}]^{\mathrm{mc}}\right)-[\check{\mathrm{S}}]^{\mathrm{mc}}\left[E_{0}\right] k_{2}}{v\left(\frac{v}{k_{2}}-\left[E_{0}\right]\right)}
$$

Negative molar mass is unusual and so, in Eq. (41), $[\check{S}]^{\mathrm{mc}}\left[E_{0}\right] k_{2}$ must be $>v\left(K_{\mathrm{m}}^{\mathrm{mc}}+[\check{S}]^{\mathrm{mc}}\right)$. However, the same cannot be said of Eq. (28-30). In the equations the unit of mass in the denominator and nominator cancel out. Therefore, there is no question of making any quantity of matter per mole subject of the formula. Unlike Eq. (1a), Eq. (28-30) can be used to estimate the time it may take to produce a desired concentration of reducing sugar given a specified concentration of the substrate and enzyme under a given condition of assay. The time needed to hydrolyze a desired amount of substrate given initial concentration can also be estimated. Unlike Eq. (1a), Eq. (28-30) can enable one to switch from pseudo-first order rate constant $k$ to first order rate constant $k_{2}$ and vice versa.

The quantitative results obtained are shown in Table 1 . The values of exp $\left(k_{\text {Qua }} t\right)$ calculated from the quadratic equation (Eq. (34b)) was not significantly different $(P>0.05)$ from the values of $\exp (k t)$ and $\exp \left(k_{\mathrm{c}} t\right)$ in which $k$ and $\exp \left(k_{\mathrm{c}} t\right)$ is the pseudo-first order rate constant determined from the plot of $\operatorname{In} \frac{\left[S_{0}\right]}{[S]}$ versus time $(t)$ and $\frac{\left[S_{0}\right]}{[S]}$ respectively. The value of $k_{2(\text { Qua) }}$ and $k_{2}$ (the rate constant for the formation of product, maltose) obtained from Eq. (29 or 30$)$ and $v_{\max } /\left[E_{0}\right]$ respectively are not statistically significant $(P>0.05)$. Also, the differences in the result between $\Delta\left[S_{0}\right]\left(\left[S_{0}\right]-324[P]\right)$ and $\left[S_{0}\right]\left(1-\frac{1}{\exp (k t)}\right)$ was not significant $(\mathrm{P}>$ 0.05 ). Given the equation $[P]$ (or $v t)=k\left[S_{0}\right] t$, the value of $[P]$ in $\mathrm{g} / \mathrm{L}$ will appear to be indefinite with different duration of assay or any enzyme activity: But this is unlike the derived quadratic or unification equations as the case may be, Eq. $(22,28-30,36)$ and the usual or conventional equation, Eq. (3).

The rate constant $\left(k_{2}\right)$ otherwise called turn over number is determined at different temperatures by many investigators [17] in order to determine the kinetic and thermodynamic characteristic of the enzymes. But it has been made known that there are different aspects of the process involved in the amylolytic production of reducing sugar, namely transit of enzyme towards the heavier polysaccharide, catalytic orientation, hydrolysis of the glycosidic bonds and making of bond and the departure of product [22]. Each aspect takes some time and, in this regard, the hydrolysis of the bond and making of new bond cannot be an exception. The rate constant for the breaking and making of bond is the main objective of this research and the result shows that it is slightly $<1 / 2^{\text {th }}$ the value of $k_{2}$.

Any means including genetic engineering that alters the physicochemical characteristic of the active site could alter the rate of bond breaking and making thereby either increasing or decreasing the value of $\boldsymbol{k}_{2[\mathrm{~s}]^{\prime}}$. It represents a useful way of controlling the overall rate of the production of simple sugars and consequently blood sugar level in diabetics in particular. This is where the pancreatic alpha amylase becomes very relevant. On the other hand in clinical and scientific research, human stress testing for instance requires the amylolytic action of human salivary alpha amylase. The most important issue is the parameter $\boldsymbol{k}_{2[S]}$ that may be seen as the rate limiting factor. This factor should be seen to be important in the clearance of starch or modified starch from starch or modified starch based encapsulating agents [41-43] for therapeutic drug delivery to target tissue and organ. Although $k_{2}$ may appear to be sufficient in monitoring the rate of production of reducing sugar, but the means to an end could be more important. "Without master card or verb card it is impossible for now to get money from automated teller machine (ATM). One might get to the ATM at the speed of light, but the person cannot control the network that determine the speed at which the machine processes the request expressed via the ATM card before the grant is made and cash ejected, the speed of which, may be fast". 
Table 1: Calculated parameters viz $\Delta\left[S_{0}\right], \exp (k t), k_{2[\mathrm{~S}]}$ and $k_{2}$

\begin{tabular}{|c|c|c|c|c|c|c|}
\hline \multirow{2}{*}{ Parameters } & \multicolumn{6}{|c|}{ Time/min } \\
\hline & 0.67 & 1.00 & 1.50 & 2.00 & 2.50 & 3.00 \\
\hline$\Delta\left[S_{0}\right] / \mathrm{g} / \mathrm{L}(324[P])$ & $0.680 \pm 0.044$ & $1.129 \pm 0.014$ & $1.502 \pm 0.007$ & $1.965 \pm 0.051$ & $2.177 \pm 0.037$ & $2.455 \pm 0.042$ \\
\hline$\chi\left[S_{0}\right] / \mathrm{g} / \mathrm{L}$ & $0.549 \pm 0.008$ & $0.807 \pm 0.012$ & 1.1830 .043 & $1.551 \pm 0.021$ & $1.900 \pm 0.025$ & $2.236 \pm 0.107$ \\
\hline $\operatorname{Exp}(k t)$ & $1.058 \pm 0.001$ & $1.088 \pm 0.001$ & $1.136 \pm 0.002$ & $1.184 \pm 0.003$ & $1.235 \pm 0.004$ & $1.288 \pm 0.005$ \\
\hline $\operatorname{Exp}\left(k_{c} t\right)\left(=\frac{\left[S_{0}\right]}{[S]}\right)$ & $1.088 \pm 0.001$ & $1.128 \pm 0.001$ & 1.1770 .001 & $1.244 \pm 0.007$ & $1.278 \pm 0.006$ & $1.325 \pm 0.007$ \\
\hline $\exp \left(k_{\text {Qua }} t\right)$ & 1.088 & 1.128 & 1.177 & 1.244 & 1.278 & 1.325 \\
\hline $\boldsymbol{k}_{2[\mathrm{~S}]}(1 / \mathrm{min})$ & \multicolumn{6}{|c|}{$9202.74 \pm 119.65$} \\
\hline$k_{2}(1 / \mathrm{min})$ & \multicolumn{6}{|c|}{18604.7286 .50} \\
\hline$k_{2(\text { Qиа) }}(1 / \mathrm{min})$ & \multicolumn{6}{|c|}{18468.382754 .25} \\
\hline
\end{tabular}

The Michaelis-Menten constant $\left(K_{\mathrm{m}}\right)=9.009 \pm 0.068 \mathrm{~g} / \mathrm{L}$; The maximum velocity of hydrolysis $\left(v_{\max }\right)=14389 \pm 67 \mathrm{U} / \mathrm{mL} ; v_{\max (\text { Qua })}=14284 \pm 2130 \mathrm{U} / \mathrm{mL}(1 \mathrm{U}$ $=$ micromoles maltose released $/ \mathrm{mL}$ enzyme in the reaction mixture $/ 3 \min . \operatorname{Exp}\left(k_{\text {Qua }} t\right)$ is calculated from the quadratic equation; $\chi$ is $\left(1-\frac{1}{\exp (k t)}\right) ; k$ is the pseudo-first order rate constant determined from the plot of $\operatorname{In} \frac{\left[S_{0}\right]}{[S]}$ versus time $(t)$ where $\left[S_{0}\right]$ and $[S]$ are the initial substrate concentration and substrate concentration as $t \rightarrow ;[P]$ is the molar concentration of the product, maltose; $k_{2}$ and $\boldsymbol{k}_{2[\mathrm{~S}]}$ are the rate constant for production formation and rate constant for the hydrolysis of glycosidic bond and making of new bond respectively.

\section{Conclusion}

Various equations, mainly quadratic equations, were successfully derived and the results obtained from them were similar to that obtainable from the conventional equations. However, the difference in the result between $\Delta\left[S_{0}\right]\left(\left[S_{0}\right]-\right.$ $324[P])$ and $\left[S_{0}\right]\left(1-\frac{1}{\exp (k t)}\right)$ and between the value of $k_{\text {2(Qua) }}$ (the constant from quadratic equation ) and $k_{2}$ (the rate constant from conventional equation) were not statistically significant $(P>$ $0.05)$. Also, there was no significant difference $(P>0.05)$ between the values of exp $\left(k_{\text {Qua }} t\right)$ calculated from the quadratic equation (Eq.(34b)) and the values of exp $(k t)$ and $\exp \left(k_{\mathrm{c}} t\right)$ in which $k$ and $\exp \left(k_{c} t\right)$ is the pseudo-first order rate constant determined from the plot of In $\frac{\left[S_{0}\right]}{[S]}$ versus time $(t)$ and $\frac{\left[S_{0}\right]}{[S]}$ respectively. The main objective, the determination of the rate constant for the breaking and making of bond was accomplished and the result showed that the value is about $1 / 2^{\text {th }}$ the value of $k_{2}$ leaving one to conclude that the limiting step may be the hydrolysis of the glycosidic bond and making of new bond.

\section{Acknowledgement}

None.

\section{Conflict of Interest}

There is neither any financial interest nor any conflict of interest.

\section{References}

1. Butterworth PJ, Warren FJ, Grassby T, Patel H, Ellis PR. (2012) Analysis of starch amylolysis using plots for first-order kinetics. Carbohydrate Polymers 87(3): 2189-2197.

2. Stoisser T, Brunsteiner M, Wilson DK, Nidetzky B (2016) Conformational flexibility related to enzyme activity: evidence for a dynamic active-site gatekeeper function of Tyr215 in Aerococcus viridans lactate oxidase. Sci Rep 6: 27892.

3. Brooks HB, Davidson VL (1993) A Method for extracting rate constants from initial rates of stopped-flow kinetic data: application to a physiological electron-transfer reaction. Biochem J 294(1): 211-213.

4. Christensen T, Stoffer BB, Svensson B, Christensen U (1997) Some details of the reaction mechanism of glucoamylase from Aspergillus niger Kinetic and structural studies on Trp52dPhe and Trp317hPhe mutants. Eur J Biochem 250(3): 638-645.

5. Ewers D, Becher T, Machtens JP, Weyand I, Fahlke C (2013) Induced fit substrate binding to an archeal glutamate transporter homologue. Proc Natl Acad Sci USA 110(30): 12486-12491.

6. de Souza PM, e Magalhães PO (2010) Application of microbial alpha amylase in industry-a review. Braz J Microbiol 41(4): 850-861.

7. Ruiz MI, Sanchez CI, Torrres RG, Molina DR (2011) Enzymatic hydrolysis of cassava starch for production of bioethanol with a Colombian wild yeast strain. J Braz Chem Soc 22(12): 2337-2343.

8. Uthumporn U, Zaidul ISM, Karim AA (2010) Hydrolysis of granular starch at sub-gelatinization temperature using a mixture of amylolytic enzymes. Food and Byproducts Process 88(1): 47-54.

9. Jaspreet Singha J, Kaurb L, McCarthy OJ (2007) Factors influencing the physico-chemical, morphological, thermal and rheological 
properties of some chemically modified starches for food applications-A review. Food Hydrocolloids 21(1): 1-22.

10. Wu AC, Ral JP, Morell MK, Gilbert RG (2014) New Perspectives on the Role of $\alpha$ - and $\beta$-Amylases in Transient Starch Synthesis. Plos One 9(6): 1-10.

11. Jemli S, Ayadi-Zouari D, Hlima HB, Bejar S (2015) Biocatalysts: application and engineering for industrial purposes. Crit Rev Biotechnol 8(2): 1-13.

12. Xian L, Wang F, Luo X, Feng YL, Feng JX (2015) Purification and characterization of a highly efficient calcium-independent $\alpha$-amylase from Talaromyces pinophilus. PLoS one 10(3): 1-18.

13. Herrero-Martínez JM, Schoenmakers PJ, Kok WTH (2014) Determination of the amylose-amylopectin ratio of starches by iodine-affinity capillary electrophoresis. J Chromatogr A 1053(12): $227-234$.

14. Absar N Zaidul ISM, Takigawa S, Hashimoto N, Matsuura Endo C, Yamauchi H. et. al. (2008) Enzymatic hydrolysis of potato starches containing different amounts of phosphorus. Food Chemistry 112(1): 57-62.

15. Kasprzak MM, Laerk HN, Larsen FH, Knudsen KEB, Pedersen S, et al. (2012) Effect of enzymatic treatment of different starch sources on the in vitro rate and extent of starch digestion. Int J Mol Sci 13: 929-942.

16. Slaughter SL, Ellis PR, Butterworth PJ (2001) An investigation of the action of porcine pancreatic alpha amylase on native and gelatinized starches. Biochim Biophysica Acta 1525(1-2): 29-36.

17. D' Amico S, Marx JC, Gerday C, Feller G (2003) Activity - stability relationships in extremophilic enzymes. J Biol Chem 276(10): 7891-7896.

18. Ohtomo T, Igarashi S, Takagai Y (2013) Flow injection spectrophotometric analysis of human salivary $\alpha$-amylase activity using an enzyme degradation of starch-iodine complexes in flow channel and its application to human stress testing. Biol Pharm Bull 36(11): 1857-1861.

19. Feroz KA, Umma Reddy CH, Anitha M, Sudarshan C, Thanikainathan M (2016) Miracle of saliva in diagnosis of oral cancer. IJRMR 3(6): 1586-1590.

20. Read NW, Welch IM, Austen CJ, Barnish C, Bartlett CE, et al. (1986) Swallowing food without chewing; a simple way to reduce post prandial glycaemia. Br J Nutr 55(1): 43-47.

21. Udema II (2017) Unifying equation and rate constant for amylolysis of glycosidic bond. Int J Appl Life Sci Int 14(3).

22. Udema II (2016) Space-time and Entropic Characterization of Aspergillus oryzea a-amylase Catalyzed Reaction. Int J Biochem Res Rev 15(2): 1-12.

23. Reuveni S, Urbakhc M, Klafterc J (2014) Role of substrate unbinding in Michaelis-Menten enzymatic reactions. Proc Natl Acad Sci USA 111(12): 4391-4396.

24. Cipolla A, Delbrassine F, Da Lage JL, Feller G (2012) Temperature adaptations in psychrophilic, mesophilic and thermophilic chloride - dependent alpha-amylases. Biochemie 94(9): 1943-1950.

25. Sarabia-Sanchez MJ, Sanchez-Munoz MA, Perez-Velazquez, ValdezSolana MA, Avitia-Dominguez C, et al. (2017) Modes of inhibition of human protein-tyrosine phosphatase $1 \mathrm{~B}$ and aldose reductase by Moringa oleifera lam leaves extract. MOJ Biorg Org Chem 1(1): 00005
26. Tzafriri AR (2003) Michaelis-Menten kinetics at high enzyme concentrations. Bull Math Biol 65(6): 1111-1129.

27. Schnell S, Maini PK (2000) Enzyme kinetics at high enzyme concentration. Bull Math Biol 62(3): 483-499.

28. Goldstein A (1944) The mechanism of enzyme-inhibitor-substrate reactions. J Gen Physiol 27(6): 529-580.

29. Cha S, Cha CJM (1965) Kinetics of cyclic enzyme systems. Mol Pharmacol 1(2): 178-189.

30. Jericevi Ž, Kuŝter $\check{Z}$ (2005) Non-linear optimization of parameters in Michaelis-Menten kinetics. Croat Chem Acta 78(4): 519-523.

31. Baici A (2006) Enzyme kinetics: the velocity of reactions. Biochem Jp. 1-3.

32. Copeland RA (2002) Enzymes: A practical introduction to structure, mechanism, and data analysis. ( $\left.2^{\text {nd }} e d n\right)$, John Wiley \& Sons, New York, USA, pp. 1-391.

33. Marchal LM Jonkers J, Franke JTh, de Gooijer CD, Tramper J (1999) The effect of process conditions on the alpha amylolytic hydrolysis of amylopectin potato starch: An experimental design approach. Biotechnol Bioeng 62(3): 348-357.

34. Bérangère F, Laurent P, Agnes P, Claude-Gilles D (2012) Methodology for bioprocess analysis: Mass balances, yields and stoichiometries, stoichiometry and research-The importance of quantity in biomedicine. In: Innocenti A (Eds.), InTech, Croatia, China, p. 1-28.

35. Beard DA, Liang SD, Quian H (2002) Energy balance for the analysis of complex metabolic net works. Biophys J 83(1): 79-81.

36. Udema II (2017) Substrate mass conservation in enzyme catalyzed amylolytic activity. Int J Biochem Res Rev 18(1): 1-10.

37. Udema II (2016) Derivation of kinetic parameter dependent model for the quantification of the concentration and molar mass of an enzyme in aqueous solution: A case study on Aspergillus oryzea alpha-amylase. J Sci Res Reports 10(3): 1-10.

38. Bernfeld P (1955) Amylases, alpha and beta. Methods. Enzymol 1: 49-152.

39. Lineweaver H, Burk D (1934) The determination of enzyme dissociation constants. J Am Chem Soc 56(3): 658-666.

40. Eisenthal R, Cornish-Bowen A (1974) The direct linear plot: A new graphical procedure for estimating enzyme kinetic parameters. Biochem J 139(3): 715-720.

41. Balmayor ER, Tuzlakoglu K, Marques AP, Azevedol HS (2008) A novel enzymatically-mediated drug delivery carrier for bone tissue engineering applications: combining biodegradable starch-based micro-particles and differentiation agents. J Mater Sci: Mater Med 19(4): 1617-1623.

42. Desai KGH, Park HJ (2005) Recent developments in microencapsulation of food ingredients. Drying Technology 23: 1361-1394.

43. Raj V, Prabha G (2016) Synthesis, characterization and in vitro drug release of cisplatin loaded Cassava starch acetate-PEG/gelatin nanocomposites. J Assoc Arab Univ Basic Appl 21: 10-16. 